\title{
Preface to the SPECIAL ISSUE: Excitonic Solar Cells (I)
}

Search and development of clean sustainable energy or renewable energy become an imperative demand beyond the concerns of limited resource and cost of fossil fuels that our modern life is so much dependent on. Among all the renewable energy, solar energy is the most abundant and likely to make the most significant contribution to the clean energy efforts. Although the usage of solar energy has been pervasive and many technologies have been explored and developed, solar to electric energy conversion technology is arguably the most convenient and readily adaptable to couple with the modern technologies including smart grid, electric vehicles, electronic appliances, and portable electronics. It makes easy to perceive the great commercial success the classical silicon solar cells enjoyed and the application of other heterojunction tandem cells widely used in satellites and space stations. In spite of the high power conversion efficiency and ever downward manufacturing cost of such heterojunction solar cells, their long energy payback time or high energy-consumption in material processing and device fabrication has humbled their domination of solar cell market and seriously retarded their penetration in the vast clean energy field.

Excitonic solar cells (ESCs) including dye-sensitized solar cells (DSCs), quantum solar cells (QDSCs), perovskite solar cells (PSCs), and organic photovoltaics (OPVs), have emerged as an alternate approach to complement the heterojunction solar cells, attracted an increasing attention, and demonstrated a promising progress with high power conversion efficiency at economically viable cost with low energy manufacturing processes. The sudden rise and rapid and dazzling progress in PSCs in the past years have infused new energy to the research field of solar cells and attracted many scientists, engineers and graduate students to the fields. New breakthroughs, new materials, innovative synthesis and processing methods, smart device design and fabrication procedures, and better in-depth fundamental understanding have been mushroomed and published in a wide variety of journals and national and international conferences. This special issue on Excitonic Solar Cells (I) is focused on the recent development and fundamental understanding of PSCs to provide the readers a relatively comprehensive overview and at the same time to promote the research on PSC.

Organic-inorganic halide PSCs have recently attracted enormous attention for thin-film solar cells due to high optical absorption coefficient, long free carrier diffusion length and low exciton binding energy. To date, the highest power conversion efficiency of PSCs that based on low temperature solution-process above $22 \%$ have been achieved, approaching the record efficiencies of monocrystalline silicon-based solar cell (25.6\%) and thin film single-crystalline GaAs cells (28.8\%). The amazing performance makes the PSC become rising star in the photovoltaic solar field. Zhao et al. reviewed the recent progress and applications in the development of PSCs.

At present, most of the highly efficient PSCs employ architecture of electron transporting layer (ETL)/perovskite absorber/hole transporting layer (HTL). PSCs contain several key interfaces, including electrode/electron transport materials (ETM) interface, ETM/perovskite interface, perovskite/hole transport materials (HTM) interface, HTM/electrode interface. The interface is vital to the overall performance of the devices, since the exciton formation, dissociation, and recombination are directly related to the interface. Wang and Yang et al. reviewed the interface engineering and modification in PSCs.

For PSCs, the inorganic metallic oxides (e.g., $\mathrm{TiO}_{2}$ and $\mathrm{ZnO}$ ) are usually chosen as the ETL, whilst some organic polymers and molecules (e.g., spiro-OMeTAD) are introduced as HTL. Here, Li et al. designed an efficient hole transporting layer free PSC. Tian and Cao et al. optimized $\mathrm{TiO}_{2}$ ETL prepared by sol-gel for high efficiency PSCs. In addition, Han et al. provided a review of n-type metal-oxide electron transport layer for mesoscopic PSCs.

Recently, lead halide perovskite colloidal quantum dots (QDs) also have attracted broad research interest in solar cells, light emitting diodes (LED), lasing, and photodetectors application because of their broad band absorption, high photoluminescence (PL) quantum yield up to $90 \%$, narrow PL emission bandwidth, spectrally tunable emission across the whole visible range tuned by simply changing the composition and QD sizes, favorable optical 
gain and low-threshold spontaneous emission. Zheng et al. studied the photo-stability of $\mathrm{CsPbBr} 3$ perovskite QDs for optoelectronic application.

Though PSCs have reached high power conversion efficiency, challenges including the hysteresis of photocurrent density-voltage and the instability of perovskite, remain to be solved for their wide-spread practical applications for the solar energy harvesting. Perovskite with new chemical composition may offer desired stability without hysteresis. Surface modification or engineering may eliminate the hysteresis and new device architecture and package may also provide solutions to circumvent the instability.

\section{Guest editors}

Prof. Jianjun Tian Advanced Material and Technology Institute, University of Science and Technology, China Email: tianjianjun@mater.ustb.edu.cn

Prof. Meicheng Li School of Renewable Energy, North China Electric Power University, China Email: mcli@ncepu.edu.cn

Dr. Kaibo Zheng Division of Chemical Physics, Lund University, Sweden Email: kaibo.zheng@chemphys.lu.se 